GUTPA/95/11/1

\title{
Electroweak Baryogenesis in the Next to Minimal Supersymmetric Model
}

\author{
A. T. Davies, C. D. Froggatt and R. G. Moorhouse \\ Department of Physics and Astronomy, \\ University of Glasgow \\ Glasgow G12 8QQ, U.K.
}

\begin{abstract}
In the electroweak phase transition there arises the problem of baryon number washout by sphaleron transitions, which can be avoided if the phase transition is strongly enough first order. The minimal supersymmetric standard model has just two Higgs doublets $H_{1}$ and $H_{2}$, while the next to minimal model, NMSSM, has an additional singlet, $\mathrm{N}$, this latter giving rise to the helpful feature that the Higgs potential contains a tree level trilinear field term. We use the tunneling criterion for the existence of a first order electroweak phase change. A quantitative statistical analysis indicates that with parameters of the NMSSM satisfying the experimental constraints a strong first order phase change occurs in about $50 \%$ of cases.
\end{abstract}

\footnotetext{
${ }^{1}$ Research partially funded by the UK Particle Physics and Astronomy Research Council
} 


\section{Introduction}

It is well known that there is difficulty in sustaining the hypothesis [1] of baryogenesis at the electroweak phase transition in the minimal standard model. In this standard model one Higgs doublet case the source of CP violation is the CKM quark mixing matrix which is too small to explain the observed baryon to entropy ratio. Also, requirements on the phase transition (in a perturbative treatment) seem to lead to a Higgs mass smaller than the experimental lower limit [2, 3]. To overcome these difficulties, attention has been given to extensions of the minimal Standard Model, involving the addition of extra scalars [3, 4, 5, 6, 7, 8]. Prominent among these is the extension to two Higgs doublets; other rather natural such extensions, necessitating two Higgs doublets, are supersymmetric theories. In the minimal supersymmetric standard model, MSSM, the Higgs sector is just two doublets [9, 10, 11, 12, 13. As in the standard model the requirement on the electroweak phase transition is that it be first order so as to avoid washout of the baryon asymmetry immediately after the transition; it appears difficult to avoid this baryon washout in the MSSM [12].

Here we shall discuss the next-to-minimal model, NMSSM, which has additionally one singlet Higgs scalar [9, 10]. Our treatment is perturbative; we do not treat the non-perturbative ideas which it has been suggested [14] might rehabilitate baryogenesis in the minimal, one Higgs doublet, Standard Model; and might when fully established provide a more secure basis for judgement on the various models. Additionally to the case of electroweak baryogenesis, it is important to examine the nature of the electroweak phase transition because of the many theories which do not create a net B-L in a phase change at higher energy; such theories are also menaced by baryon washout.

In the absence of hard information we have to adopt a hypothesis on the SUSY breaking scale and on the spectrum of the particles. We follow a number of papers of recent years in taking the SUSY breaking scale, $M_{S}$, to be of the order of $1 \mathrm{TeV}$; we take perfect supersymmetry above that scale [15, 16]. Then at $M_{S}$ the quartic scalar couplings are fixed by the gauge couplings and two more parameters. We then use the renormalization group (RG) equations to run down the quartic couplings to the electroweak scale, where we investigate the nature of the phase change. There are also cubic and quadratic supersymmetry breaking couplings, and there results a 
space of variable parameters in which we investigate what proportion leads to a strongly first order electroweak phase change, and so is compatible with electroweak baryogenesis.

There has been quite considerable previous work on the electroweak phase change in the MSSM. We are not aware of so much on the NMSSM . The work of Pietroni [17] has pointed up that the NMSSM, in contrast to the MSSM, has cubic terms in the scalar field potential at tree level leading to the possibility of a potential barrier in radial directions even at tree level; and that this diminishes the importance of the $m^{3} T$ term, in the high-T expansion of the T-dependent part, which has a vital role in most other theories of the phase change. That work uses a unitary gauge which we consider to be not so secure a basis for the consideration of phase changes as the Landau gauge which we use [8, 18].

A further difference is that we include the $\mu$-term in the superpotential of the NMSSM; $\mu=0$ is incorporated as a special case. Though there is a known naturalness problem with the magnitude of $\mu$, the often preferred solution of setting $\mu$ equal to zero raises cosmic domain wall problems [19]. When $\mu \neq 0$ one often used [8, 12, 17] definition of the critical temperature, being that temperature at which the curvature of the potential at the origin vanishes, can no longer be justified.

We adopt the alternative definition, being that temperature when tunneling first becomes possible from the high- $T$ vacuum to the low- $T$ vacuum having non-zero Higgs doublets expectation values. This criterion being wellknown and discussed [1, 20, has on occasion actually been used in calculations [21], though it is more difficult to implement than the curvature criterion. This is an important change from most previous calculations, such as those of Ref. [8, 12] in the MSSM and Ref. [17] in the NMSSM. Also this paper has the new feature that it compares the results from the two criteria when both can be implemented, that is in the $\mu=0$ case.

\section{Formalism of the NMSSM}

The magnitudes of the parameters of the superpotential are significant for the electroweak phase transition. With the usual notation for the quark and lepton superfields, and summation over generations understood, the super- 
potential is

$$
\begin{aligned}
W= & g_{u} Q u^{c} H_{2}+g_{d} Q d^{c} H_{1}+g_{e} L e^{c} H_{1} \\
& +\mu H_{1} H_{2}+\lambda H_{1} H_{2} N-\frac{k}{3} N^{3}-r N
\end{aligned}
$$

where $H_{1}, H_{2}$ are the Higgs doublet, and $N$ the singlet, superfield 2. The corresponding tree level scalar field potential, below the supersymmetry breaking scale, is [9, 10]

$$
\begin{aligned}
V_{0}= & \frac{1}{2}\left(\lambda_{1}\left(H_{1}^{\dagger} H_{1}\right)^{2}+\lambda_{2}\left(H_{2}^{\dagger} H_{2}\right)^{2}\right)+ \\
& \left(\lambda_{3}+\lambda_{4}\right)\left(H_{1}^{\dagger} H_{1}\right)\left(H_{2}^{\dagger} H_{2}\right)-\lambda_{4}\left|H_{1}^{\dagger} H_{2}\right|^{2}+ \\
& \left(\lambda_{5} H_{1}^{\dagger} H_{1}+\lambda_{6} H_{2}^{\dagger} H_{2}\right) N^{\star} N+\left(\lambda_{7} H_{1} H_{2} N^{\star 2}+\text { h.c. }\right)+ \\
& \lambda_{8}\left(N^{\star} N\right)^{2}+\left(|\mu|^{2}+\left(\lambda \mu^{\star} N+\text { h.c. }\right)\right)\left(H_{1}^{\dagger} H_{1}+H_{2}^{\dagger} H_{2}\right)+ \\
& m_{1}^{2} H_{1}^{\dagger} H_{1}+m_{2}^{2} H_{2}^{\dagger} H_{2}+m_{3}^{2} N^{\star} N-\left(m_{4} H_{1} H_{2} N+\text { h.c. }\right)- \\
& \frac{1}{3}\left(m_{5} N^{3}+\text { h.c. }\right)+\frac{1}{2}\left(m_{6}^{2} H_{1} H_{2}+\text { h.c. }\right)+\left(m_{7}^{2} N^{2}+\text { h.c. }\right)
\end{aligned}
$$

where

$$
H_{1}{ }^{T}=\left(H_{1}^{0}, H_{1}{ }^{-}\right), H_{2}{ }^{T}=\left(H_{2}{ }^{+},{H_{2}}^{0}\right), H_{1} H_{2}=H_{1}{ }^{0} H_{2}{ }^{0}-H_{1}{ }^{-} H_{2}{ }^{+}
$$

$H_{1}, H_{2}$ and $N$ now denoting pure scalar fields. The $m$-coefficient terms comprise all possible soft supersymmetry breaking terms [9, 10]. $V_{0}$ is a function of 10 real scalar fields, the Higgs doublets and the singlet being given in terms of these by

$$
H_{1}=\frac{1}{\sqrt{2}}\left(\begin{array}{c}
\phi_{1}+i \phi_{2} \\
\phi_{3}+i \phi_{4}
\end{array}\right), H_{2}=\frac{1}{\sqrt{2}}\left(\begin{array}{c}
\phi_{5}+i \phi_{6} \\
\phi_{7}+i \phi_{8}
\end{array}\right), N=\frac{1}{\sqrt{2}}\left(\phi_{9}+i \phi_{10}\right)
$$

For simplicity, and to automatically ensure real VEVs, we shall follow the usual practice and take the parameters real. The boundary values at $M_{S}$ of the quartic couplings are given by [22]

$$
\lambda_{1}=\lambda_{2}=\frac{1}{4}\left(g_{2}^{2}+g_{1}^{2}\right), \lambda_{3}=\frac{1}{4}\left(g_{2}^{2}-g_{1}^{2}\right), \lambda_{4}=\lambda^{2}-\frac{1}{2} g_{2}^{2},
$$

\footnotetext{
${ }^{2}$ A possible term $N^{2}$ in the superpotential can be removed by a field redefinition 10 .
} The linear term, $r N$, contributes terms to the effective potential of the same type as we include with arbitrary coefficients in the soft supersymmetry-breaking part of the potential $V_{0}$ given in Eq. (2). 


$$
\lambda_{5}=\lambda_{6}=\lambda^{2}, \lambda_{7}=-\lambda k, \lambda_{8}=k^{2}
$$

and are developed down to $M_{\text {Weak }}$ by using the appropriate RG equations [22]. The $\lambda$ and $\mathrm{k}$ are in principle free parameters at $M_{S}$ [9, 10. However they are linked to the one important Yukawa coupling [ $^{2} g_{t}$, by 3 simultaneous RG equations 23] in developing from high energy down to $M_{S}$; their values there should not be such that they correspond to divergent or unnaturally large values at high energy. From Eqs. (2) and (3) we obtain the zero temperature potential $V_{0}(\phi)$ as a function of $\phi=\phi_{1}, \phi_{2}, \ldots, \phi_{10}$ with the parameters $\lambda_{i}$ assumed renormalised at the electroweak scale. The vacuum expectation values of the scalars are to be found at the minimum of $V_{0}$ and thus satisfy

$$
\left.\partial_{i} V_{0}\right|_{\phi=\langle\phi\rangle}=0, \quad \partial_{i} \equiv \frac{\partial}{\partial \phi_{i}}, i=1,2, \ldots, 10
$$

and also the requirement that $\langle\phi\rangle$ be a minimum.

It is a constraint on our parameters that the minimum of $V_{0}$ at zero temperature is for the VEVs of the Higgs fields having neutral components only

$$
\left\langle H_{1}\right\rangle=\left(\begin{array}{c}
v_{1} \\
0
\end{array}\right),\left\langle H_{2}\right\rangle=\left(\begin{array}{c}
0 \\
v_{2}
\end{array}\right),\langle N\rangle=x
$$

where $v_{1}, v_{2}$ and $x$ are real and $v=\sqrt{v_{1}^{2}+v_{2}^{2}}=174 \mathrm{GeV}$. The scalar masssquared matrix is given by

$$
M_{i j}^{2}=\left.\partial_{i} \partial_{j} V_{0}\right|_{\phi=\langle\phi\rangle}
$$

and gives rise to 7 massive physical particles and 3 zero mass would-be Goldstone bosons. The $m_{1}^{2}, m_{2}^{2}, m_{3}^{2}$ are standard mass parameters and are to be specified in terms of the VEVs and the other parameters by Eq. (团) when $\phi$ is given by Eq. (5).

We can now discuss the other parameters in $V_{0}$.

Firstly there are the terms involving $\mu$ which arise from the $\mu$ term in the superpotential. This raises the mu-problem(first noted in the MSSM) in the context of the NMSSM [19, 25]. Unlike the $\lambda$ and $\mathrm{k}$ parameters of the superpotential just discussed there is no control on $\mu$, which would naturally

\footnotetext{
${ }^{3}$ we are not considering large $\tan \beta$ here
} 
be expected to take on a value of the order of magnitude of the fundamental scale of the theory, whereas phenomenologically it should be of the order of the other electroweak terms. We do not take the point of view that the NMSSM can solve this by its having largely phenomenologically equivalent terms in the $\mathrm{N}$ field and simply setting $\mu=0$ 23, 25]. This can have its own difficulties when a resulting $Z_{3}$ symmetry gives rise to domain walls $19 \|$. We tolerate the mu-problem. It should be noted that we have extra $Z_{3}$ breaking by the phenomenological term $m_{6}^{2} H_{1} H_{2}$. Secondly there are the remaining soft parameters $m_{4}, m_{5}, m_{7}^{2}$; their provenance as completing the most general NMSSM breaking $V_{0}$ was given in Refs. [9, 10].

Going now to the T-dependent terms in the effective potential we have the development, by the usual methods 18 for a temperature sufficiently high compared to the masses, given by

$$
\begin{gathered}
V(\phi, T)=V_{0}(\phi)-\frac{N_{e f f} \pi^{2} T^{4}}{90}+V_{2}(\phi, T)+V_{3}(\phi, T)+V_{l n}(\phi, T) \\
V_{3}(\phi, T)=-\frac{T}{12 \pi}\left[\sum_{i=1}^{10}\left[M_{i}^{2}(\phi, T)\right]^{\frac{3}{2}}+4\left[\frac{g_{2}^{2}}{4} \sum_{i=1}^{8} \phi_{i}{ }^{2}\right]^{\frac{3}{2}}+2\left[\frac{g_{2}^{2}+g_{1}^{2}}{4} \sum_{i=1}^{8} \phi_{i}{ }^{2}\right]^{\frac{3}{2}}\right] \\
V_{l n}(\phi, T)= \pm \sum_{i} n_{i} \frac{M_{i}^{4}}{64 \pi^{2}} \ln \left[\frac{M_{i}^{2}}{c_{i} T^{2}}\right]
\end{gathered}
$$

In Eq. (9) \pm refers to fermions and bosons respectively, and $n_{i}$ is the number of degrees of freedom of the particle [5].

$$
V_{2}=\frac{T^{2}}{24} V_{\text {trace }}
$$

where $V_{\text {trace }}$ is the trace of the mass squared matrix 


$$
\begin{aligned}
V_{\text {trace }}= & 4 m_{1}^{2}+4 m_{2}^{2}+2 m_{3}^{2}+8 \mu^{2}+ \\
& \left(3 \lambda_{1}+2 \lambda_{3}+\lambda_{4}+\lambda_{5}+\frac{3}{4}\left(3 g_{2}^{2}+g_{1}^{2}\right)\right)\left(2 H_{1}^{\dagger} H_{1}\right)+ \\
& \left(3 \lambda_{2}+2 \lambda_{3}+\lambda_{4}+\lambda_{6}+\frac{3}{4}\left(3 g_{2}^{2}+g_{1}^{2}\right)+3 g_{t}^{2}\right)\left(2 H_{2}^{\dagger} H_{2}\right)+ \\
& \left(2 \lambda_{5}+2 \lambda_{6}+4 \lambda_{8}\right)\left(2 N^{\star} N\right)+8 \lambda \mu(N+\text { h.c. })+ \\
& 4|(\mu+\lambda N)|^{2}+2 \lambda^{2}\left(\left|H_{1}\right|^{2}+\left|H_{2}{ }^{0}\right|^{2}\right)+4 k^{2} N^{\star} N .
\end{aligned}
$$

The last line is the 1-loop contribution of the Higgsinos in the case where the masses of the winos and binos are taken large, of order $M_{S}$, and is the sum of their squared masses. The size of the parameters gives these masses to be significantly less than $M_{S}$ and thus they should be taken into account in the 1-loop corrections. In Eq. (8), the first term is the contribution from the scalar masses. Here we have not just used the tree level masses squared, which are eigenvalues of $\partial_{i} \partial_{j} V_{0}$ as used in Eq. (10), but we use the eigenvalues of $\partial_{i} \partial_{j}\left(V_{0}(\phi)+V_{2}(\phi, T)\right)$. It has been shown [2, 24] that this is equivalent to correcting the one-loop potential by adding to the loop all ring diagrams ('daisy diagrams') which makes what would otherwise be imaginary values of $\left[M_{i}^{2}\right]^{\frac{3}{2}}$, for some values of $\phi$, into real values [18, 20. Also in Eq. (8), a correction to the gauge boson contribution has been included; in the contribution of the longitudinal polarisation excitations, there is suppression due to a temperature dependent 'Debye mass' factor. A simple treatment of this due to Dine et al. [2] is just to drop the longitudinal contribution, and we follow this prescription.

A technical complication that arises is that if $\frac{M_{i}}{T} \geq 2.2$ for bosons or $\frac{M_{i}}{T} \geq 1.8$ for fermions then the expansion of Eq. (7) breaks down; this is important at $T=T_{\text {crit }}$. We solve this by the method of Anderson and Hall [5] and replace the last three terms of Eq. (7) by

$$
\Delta V(\phi)=-\sum_{i} \frac{n_{i} T^{2} M_{i}^{2}}{(2 \pi)^{\frac{3}{2}}} \sqrt{\frac{T}{M_{i}}} e^{-\frac{M_{i}}{T}}\left[1+\frac{15 T}{8 M_{i}}+\cdots\right]
$$

in the appropriate high $\frac{M_{i}}{T}$ region. Here $n_{i}$ is the number of degrees of freedom of particle $\mathrm{i}$. 


\section{Phase Change Calculations}

Calculations with the kinetic equations for the dilution of baryonic charge just after the phase transition give a baryon preservation condition [2, 7]

$$
\frac{v\left(T_{\text {crit }}\right)}{T_{\text {crit }}} \geq \xi
$$

where $\xi$ varies between about 0.9 and 1.5 according to the gauge and other couplings of the theory. We take $\xi=1$. In practice in various theories just two criteria have been used to find the critical temperature, $T_{\text {crit }}$, and authors have made a choice of either one or the other; we use both and compare. One is the temperature, $T_{0}$, at which, for decreasing $T$, the curvature of the effective potential $V(\phi, T)$ at $\phi=0$ (assumed to be the previous global minimum) first vanishes in the Higgs doublet neutral field directions. The other is the temperature, $T_{C}$, at which the value of $\mathrm{V}$ at a different minimum with a non-zero $v^{\prime}$ first becomes the global minimum of $V\left(v_{1}^{\prime}, v_{2}^{\prime}, x^{\prime}, T\right)$; here the prime denotes general values of the field quantities as opposed to the specific values $v_{1}, v_{2}, x$ proper for the $\mathrm{T}=0$ physical theory. As discussed below only for $\mu=0$ is the first criterion, $T_{\text {crit }}=T_{0}$, applicable. Now the shape of $V\left(v_{1}^{\prime}, v_{2}^{\prime}, x^{\prime}, T\right)$ depends on the theory parameters. It is the space of variable parameters which we search to assess in what part, and what proportion, of it the criterion Eq. (12) is satisfied. These variables are:

1. $[\mu, \lambda, k]$, occuring in the superpotential Eq. (1);

2. $\left[\tan \beta \equiv v_{2} / v_{1}, x\right]$ (together with the known fixed value of $v$ ) specify the VEVs and replace $m_{1}^{2}, m_{2}^{2}, m_{3}^{2}$;

3. $\left[M_{c h}\right]$, the mass of the charged Higgs, is similarly given by an analytic formula, and we take it to replace $m_{4}$ as a variable;

4. $\left[m_{5}, m_{6}^{2}, m_{7}^{2}\right] ; m_{6}^{2}$, when non-zero, breaks the $Z_{3}$ symmetry even in the absence of $\mu$.

It is also necessary to consider the permissible range for the top Yukawa coupling, $g_{t}$. We do not investigate the case where $\tan \beta$ is very large, this re-

striction implying that $g_{t}^{2} \gg g_{b}^{2}$; there then are 3 simultaneous RG equations for $\lambda, k, g_{t}$ [23]. These run from high energy down to $M_{S}$, where the $\lambda, k, g_{t}$ 
should not be such that they correspond to divergent or unnaturally large values at high energy. The first limitation that this induces is $g_{t}\left(M_{S}\right) \leq 1.06$ which corresponds to $g_{t}\left(m_{t}\right)<1.12$. The experimental value of $m_{t}\left(m_{t}\right)$, being greater than about $150 \mathrm{GeV}$, induces a lower limit on $g_{t}$ through the relation $m_{t}=g_{t} v \sin \beta$, where $v=174 \mathrm{GeV}$, resulting in $g_{t}\left(m_{t}\right)>0.85$.

Within the above small available range for $g_{t}$ we select $g_{t}\left(M_{S}\right)=1$ and we now discuss the relevant ranges of the above variables (i)-(iv):

1. $[\mu, \lambda, k]$ : We start by noting that the presence of $\mu$, important fundamentally, has an interesting consequence for the minimum of $\mathrm{V}$ at non-zero $\mathrm{T}$. We discuss this in the high $\mathrm{T}$ expansion. As displayed above, Eq. (10), $V_{2}$ is a function of the fields with the only linear terms being those in $(\lambda \mu N+$ h.c. $)$. This means that for any non-zero $\mathrm{T}$ the origin $\phi_{i}=0$ cannot be a turning value and so cannot be a minimum for $\mu \neq 0$. We are in a situation different from that in the MSSM but similar to that discussed by Choi and Volkas [21] in a different theory: the false vacuum is not at the origin, and the transition to the true vacuum is more complicated but amenable to investigation. It follows that only for the special case $\mu=0$ is the critical temperature criterion of vanishing curvature at the origin, $T_{\text {crit }}=T_{0}$, applicable. We have investigated the cases $\lambda \mu=0, \pm 20 \mathrm{GeV}, \pm 40 \mathrm{GeV}, \pm 60 \mathrm{GeV}$. The RG equations in $\lambda, k, g_{t}$ also limit the ranges of $\lambda$ and $k$, which we find roughly require $\sqrt{\lambda^{2}+k^{2}} / g_{t}$ to be less than about 0.8 23]. With our value $g_{t}\left(M_{S}\right)=1$ we have investigated the three cases $(\lambda, k)=(0.65$, $0.1) ;(0.1,0.65) ;(0.5,0.5)$.

2. $[\tan \beta, x]$ : The value of $\tan \beta$ is associated with that of the Yukawa coupling and the top mass through $m_{t}=g_{t} v \sin \beta$. We have investigated values of $\tan \beta$ in the range 1.6 to 3.0 in steps of 0.2 which, with our value of $g_{t}$, corresponds to a running top mass of 150 to $173 \mathrm{GeV}$. The parameter $x$ is rather free; the values considered are: $x=0.1 v, 0.5 v$, $v, 2 v$.

3. $\left[M_{c h}\right]$ : Accepting the interpretation of the CLEO $b \rightarrow s \gamma$ rate as requiring that $M_{c h}>200 \mathrm{GeV}$, we have searched in the region $200<$ $M_{c h}<400 \mathrm{GeV}$. 
4. $\left[m_{6}^{2}, m_{5}, m_{7}^{2}\right]$ The range of searches of these variables has been

$$
-6<\frac{m_{6}^{2}}{(50 \mathrm{GeV})^{2}}<6, \quad-6<\frac{m_{7}^{2}}{(50 \mathrm{GeV})^{2}}<6, \quad-1<\frac{m_{5}}{(60 \mathrm{GeV})}<1 .
$$

In the ranges indicated above for $M_{c h}, m_{5}, m_{6}^{2}$ and $m_{7}^{2}$, we have conducted a coarse-grained search on a multidimensional grid over the parameter space.

The first computing task is to restrict the parameter space to satisfy experimental constraints at $\mathrm{T}=0$. The conditions imposed are:

(i) the masses are real,

(ii) the minimum of $\mathrm{V}$ at the specified $\tan \beta, v(=174 \mathrm{GeV})$ and $x$ is a global minimum in the space $v_{1}^{\prime}, v_{2}^{\prime}, x^{\prime}$,

(iii) the charginos and neutralinos have masses greater than $45 \mathrm{GeV}$ and 30 $\mathrm{GeV}$ respectively, and

(iv) the mass of the lightest Higgs scalar is greater than $65 \mathrm{GeV}$.

We call the set of these acceptable parameter sets the basis space. These conditions on the basis space are more restrictive than those imposed in our conference report 27. This results in a much smaller basis space than in [27] and a much greater proportion of baryon preserving cases.

The experimental limitation, (iv), on the lightest Higgs mass does not severely limit the basis space; it cuts out only about $1 / 3$ of the sets which would otherwise pass into the basis space, whereas the first three conditions have a more severe effect.

\section{Results and Conclusions}

We need to find what proportion of the above basis space satisfies the baryon number preserving condition Eq. (12). We shall take this as a measure of the ease of baryon preservation in the NMSSM theory.

We have examined an initial grid of 105,840 parameter sets. Of these 1,760 , being $1.66 \%$, pass through the tests (i)-(iv) of Section 3 for producing an appropriate $\mathrm{T}=0$ mass spectrum and form the basis space. We have then tested each parameter set in that space for whether it passes the 
baryon preservation test, with the critical temperature defined as being that where tunneling from the higher temperature vacuum to the low temperature vacuum first becomes possible, as described in Section 3.

The breakdown of these numbers into sectors of different $\mu, \lambda, k$ and $x$, is given in Table 1. It is clear, both from the overall result and from the breakdown, that the condition of baryon number preservation just after the electroweak phase change does not impose a significant extra constraint on the parameters of the NMSSM from the present, statistical, point of view. Overall $50 \%$ of the basis space passes the test of baryon preservation. Despite the large number of parameter sets in the initial grid, the sample of ultimately successful cases is around 900, too small to permit a meaningful delineation of the acceptable regions of our 9-dimensional parameter space. In the particular case of the parameters $\mu, \lambda, k$ of the superpotential, Table 1 gives an indication of the variation of the successful proportion with $\lambda$ and $k$; for $\mu$ the criteria select a basis space with a very strong bias towards negative $\mu$ (relative to $x$ ) and, after that selection, the baryon preservation condition gives a rather constant proportion over different values of $\mu$ and so does not select further. The $65 \mathrm{GeV}$ lower bound we have placed on the mass of the lightest Higgs scalar is ultra-conservative, since it comes from analyses of experiments performed within the SM and the MSSM. In the NMSSM, with its extra mixing and different couplings, lower mass Higgs scalars could have escaped detection [26]. All parameter sets in our basis space yield a lightest Higgs scalar with a mass less than $120 \mathrm{GeV}$. The tendency is for lower mass lightest Higgs scalars to be associated with a greater proportion of baryon preserving cases (80\% for a mass of $70 \mathrm{GeV}$ but only $20 \%$ for $120 \mathrm{GeV}$ ).

In Table 2 we show some results for the special case $\mu=0$. Of chief interest is the comparison between the zero curvature at $\langle\phi\rangle=0$ criterion and the tunneling criterion for the critical temperature. Though there are some case by case differences the two criteria are statistically nearly the same; to our knowledge this is the first time such a detailed comparison has been made. It was found that the depth of the broken symmetry minimum relative to the value of $\mathrm{V}$ in the neighbourhood of the origin changed rapidly with temperature. As the perturbative effective potential formalism suffers from well-known problems near the origin particularly [18, 28, 29], the tunneling criterion is preferable to the frequently used curvature criterion, being less dependent on the value precisely at the origin.

Our conclusions for the case $\mu=0$ broadly agree with previous work on 
this case [17], although our formalism differs, e. g. our gauge choice produces different T-dependent contributions to the scalar mass matrix. To treat the hitherto uninvestigated case of $\mu \neq 0$, we have had to change from the vanishing curvature criterion to the tunneling criterion for the critical temperature; we have found that values of $\mu$ up to about $100 \mathrm{GeV}$ are acceptable, with well-chosen values of the other superpotential parameters $(\lambda, k)$. Unlike the Standard Model or the MSSM, the NMSSM can easily coexist with baryon number preservation. The essential difference is that cubic terms can already be present in the $\mathrm{T}=0$ potential, so that the $\mathrm{T}$-dependent terms modify but are not solely reponsible for the existence of a minimum away from the origin, $v=0$, at the critical temperature.

\section{Acknowledgements}

We should like to thank P. L. White and D. G. Sutherland for very useful and stimulating discussions. 


\section{References}

[1] V.A. Kuzmin, V.A. Rubakov and M.E. Shaposhnikov, Phys. Lett. B 155 (1985) 36 ; M.E. Shaposhnikov, Nucl. Phys. B 287 (1987) 757 ; L. McLerran, Phys. Rev. Lett. 62 (1989) 1075 ; A.G. Cohen, D.B. Kaplan and A.E. Nelson, Phys. Lett. B 245 (1990) 561 ; Nucl. Phys. B 349 (1991) 727; Annu. Rev. Nucl. Part. Sci. 43 (1993) 27.

[2] M. Dine, P. Huet, R. Leigh and A. Linde, Phys. Lett. B 283 (1992) 319; Phys. Rev. D 46 (1992) 550.

[3] A.I. Bochkarev, S.V. Kuzmin and M.E. Shaposhnikov, Phys. Rev. D 43 (1991) 369.

[4] N. Turok and J. Zadrozny, Phys. Rev. Lett. 65 (1990) 2331 ; Nucl. Phys. B 358 (1991) 471 .

[5] G.W. Anderson and L.J. Hall, Phys. Rev. D 45 (1992) 2685.

[6] M. Dine, P. Huet, R.Singleton and L. Susskind, Phys. Lett. B 256 (1991) 351.

[7] A.G.Cohen, D.B. Kaplan and A.E. Nelson, Phys. Lett. B 263 (1991) 86.

[8] A.T. Davies, C.D.Froggatt, G. Jenkins and R. G. Moorhouse, Phys. Lett. B 336 (1994) 464.

[9] J. Gunion, H.E. Haber, G.L. Kane and S.Dawson, 'The Higgs Hunter's Guide' (Addison-Wesley, Reading MA, 1990).

[10] J. Gunion and H.E. Haber, Nucl. Phys. B 272 (1986) 1.

[11] M. Dine, P. Huet and R. Singleton, Nucl. Phys. B 375 (1992) 625 ;

S. Myint, Phys. Lett. B 287 (1992) 325 ; G.F. Giudice, Phys. Rev. D 45 (1992) 3177.

[12] J.R. Espinosa, M. Quiros and F. Zwirner, Phys. Lett. B 314 (1993) 206; B 307 (1993) 106; A. Brignole, J.R. Espinosa, M. Quiros and F. Zwirner, Phys. Lett. B 324 (1994) 181.

[13] D. Comelli, M. Pietroni and A. Riotto, Nucl. Phys. B 412 (1994) 441. 
[14] G. Farrar and M.E. Shaposhnikov, Phys. Rev. Lett. 70 (1993) 283 ; (E) 71 (1993) 210 ; M.B. Gavela, P. Hernandez, J. Orloff and O. Pene, Mod. Phys. Lett. A 9 (1994) 795.

[15] R. Barbieri, M. Frigieri and F. Caravaglio, Phys. Lett. B 258 (1991) 167.

[16] C. D. Froggatt, I. G. Knowles and R. G. Moorhouse Phys. Lett. B 298 (1993) 356 .

[17] M. Pietroni, Nucl. Phys. B 402 (1993) 27.

[18] L. Dolan and R. Jackiw, Phys. Rev. D 9 (1974) 3320 .

[19] S. A. Abel, S. Sarker and P. L. White, Oxford University preprint OUTP-95-22P, hep-ph/9506359.

[20] D.A. Kirzhnitz and A. Linde, Ann. Phys. (NY) 101 (1976) 195.

[21] J. Choi and R. R. Volkas, Phys. Lett. B 317 (1993) 385.

[22] T. Elliott, S. F. King and P. L. White, Phys. Lett. B 305 (1993) 71.

[23] T. Elliott, S. F. King and P. L. White, Phys. Rev. D 49 (1994) 2435.

[24] M.E. Carrington, Phys. Rev. D 45 (1992) 2933 ; P. Arnold, Phys. Rev. D 46 (1992) 2682 ; J.R. Espinosa, M. Quiros and F. Zwirner, Phys. Lett. B 314 (1993) 206.

[25] J. Ellis, J. Gunion, H.E. Haber, L. Roszkowski and P. Zwirner, Phys. Rev. D 39 (1989) 844.

[26] S. F. King and P. L. White, Oxford University preprint OUTP-95-31P, hep-ph/9508346.

[27] A.T. Davies, C.D.Froggatt, G. Jenkins and R. G. Moorhouse, GUTPA/95/10/2 ( to be published in the Proceedings of the International Europhysics Conference on High Energy Physics, Brussels, 1995).

[28] P. Arnold and O. Espinosa, Phys. Rev. D 47 (1993) 3546.

[29] K. Kajantie, K. Rummukainen and M. Shaposhnikov, Nucl. Phys. B 407 (1993) 356. 
Table 1: Showing the proportion of the basis space of parameter sets which satisfy the $\mathrm{B}$ ( baryon)-preservation criterion, as a function of $\lambda, k$ and of the $\mathrm{T}=0$ value of $x(\equiv\langle N\rangle)$. In the basis space the $\mathrm{T}=0$ criteria are satisfied. The values of $\mu$ covered are given by $60 \mathrm{GeV}>\lambda \mu>-60 \mathrm{GeV}$.

\begin{tabular}{|cc|c|c|}
\hline$\lambda$ & $k$ & $x / v$ & $\begin{array}{c}\text { B preserving } \\
\text { \%-age of basis }\end{array}$ \\
\hline & & 0.1 & 40 \\
0.65 & \multirow{2}{*}{0.1} & 0.5 & 66 \\
& & 1.0 & 82 \\
& & 2.0 & 59 \\
\hline 0.1 & 0.65 & 1.0 & 50 \\
\hline 0.5 & 0.5 & 1.0 & 26 \\
\hline
\end{tabular}

Table 2: $\mu=0$ : Comparing the results from the criterion of vanishing curvature of $\mathrm{V}$ at $\langle\phi\rangle=0, \mathrm{~T}_{\text {crit }}=\mathrm{T}_{0}$, with the tunneling criterion, $\mathrm{T}_{\text {crit }}=\mathrm{T}_{\mathrm{C}}$. The baryon preserving proportion of the basis space and the average value of the critical temperature, $\mathrm{T}_{\text {crit }}$, are shown for both cases .

\begin{tabular}{|ccc|c|c|c|c|}
\hline$\lambda$ & $k$ & $x / v$ & $\begin{array}{c}\text { B preserving } \\
\% \text {-age for } \\
T_{\text {crit }}=T_{0}\end{array}$ & $\begin{array}{c}\text { Average of } \\
\mathrm{T}_{\text {crit }}=\mathrm{T}_{0}\end{array}$ & $\begin{array}{c}\text { B preserving } \\
\% \text {-age for } \\
T_{\text {crit }}=T_{C}\end{array}$ & $\begin{array}{c}\text { Average of } \\
\mathrm{T}_{\text {crit }}=\mathrm{T}_{\mathrm{C}}\end{array}$ \\
\hline 0.65 & 0.1 & 1.0 & 80 & $70 \mathrm{GeV}$ & 79 & $71 \mathrm{GeV}$ \\
\hline 0.5 & 0.5 & 1.0 & 16 & $82 \mathrm{GeV}$ & 11 & $80 \mathrm{GeV}$ \\
\hline
\end{tabular}

